\title{
Putting the Cart before the Horse: Outcomes Following Rapid Implementation of Telepsychiatry in an Outpatient Resident Clinic
}

\author{
Samar McCutcheon ${ }^{1}$ \\ Received: 11 June 2020 / Accepted: 8 September 2020 / Published online: 17 September 2020 \\ (C) Academic Psychiatry 2020
}

Historically, telepsychiatry has most often been instituted as a method to expand access of psychiatric services to patients, primarily in rural areas with a paucity of psychiatric services or as a means for patients to gain access to subspecialty psychiatric care [1-3]. In those contexts, previous studies of telepsychiatry have demonstrated similar efficacy and patient satisfaction with video visits when compared with face-to-face encounters [4-6]. Systemic reviews have established that few residency programs have formal, longitudinal telepsychiatry curriculums, but telepsychiatry education has historically been an area of interest within residency programs; up to $85 \%$ of residency programs responding to a telepsychiatry survey by Hoffman and Kane expressed interest in a sample curriculum [7-9]. The Accreditation Council for Graduate Medical Education (ACGME) does not have specific requirements surrounding telemedicine in the psychiatry common program requirements, but it has been posited that exposure to telepsychiatry during training could increase resident competence and comfort with providing care utilizing this modality after graduation [10,11].

\section{Implementation of Telepsychiatry}

With the adjustments to patient care made in the face of the COVID-19 pandemic, telepsychiatry shifted overnight from a topic touched upon briefly in didactics at our program to the default method of practicing outpatient psychiatry in our resident clinic. We are aware that this was the shared experience of several psychiatry residency programs across the nation, facilitated by the loosening of pre-existing federal and state

Samar McCutcheon

samar.mccutcheon@osumc.edu

1 The Ohio State University College of Medicine, Columbus, $\mathrm{OH}$, USA regulations that had often served as barriers to telepsychiatry [12]. Ideally, extensive education and thorough training proceed the introduction of any new practice delivery method in residency training. If implementing telepsychiatry had been a planned transition, attendings would have reviewed the existing literature of evidence-based practices and piloted the method; residents would then be introduced to the process with background reading and lectures, transitioning to practice once attendings were capable and competent to supervise. With COVID-19, our residency was forced to put the "cart" of patient care before the "horse" of education and training.

On March 17, 2020, all scheduled outpatient visits in postgraduate year 3 (PGY-3) resident clinic at The Ohio State University Wexner Medical Center were converted from face-to-face encounters to telephone visits. Telephone visits were the standard as the medical center worked to obtain sufficient telemedicine licenses and webcams to outfit each computer. On April 6, 2020, these requirements were met, and the ability to schedule video visits was introduced. Subsequently, between March 17, 2020 and June 5, 2020 (the date of survey distribution), 1960 outpatient follow-up appointments and 94 new patient assessments were completed via telephone or video by residents within the outpatient clinic. A portion of patients, primarily within our psychosis specialty clinic, continued to have in-person appointments if the resident and attending determined the clinical benefit outweighed perceived risk; this accounted for a very small percentage of total visits $(<$ $1 \%$ ). Telephone visits continued if the patient did not have access to the Internet or declined a video visit, and in session, video visits were converted to telephone encounters if patients or residents experienced technical difficulties. This experience with telephone and video encounters was largely consistent with a recent survey of outpatient psychiatrists implementing telemedicine during COVID-19 [13].

The overnight change to telepsychiatry also impacted the supervision processes in our clinic. Before the transition, residents would assess patients in their office, step out to staff the 
case with an on-site attending, and the resident and attending would then return to the resident's office together to complete the session. With initial implementation of telepsychiatry, the existing model of supervision was continued. This required the resident and attending to don a mask when in the resident office together to complete the encounter. It was more difficult for patients to hear the treatment plan as a result and more challenging to demonstrate empathic facial expressions when wearing masks. With further understanding of the telemedicine platform (Vidyo), attendings were able to conference into video visits from their private offices, allowing both the resident and attending to remain mask-free throughout the encounter and improving the audio for patients.

With this rapid shift from in-person to virtual visits, communication with residents centered on dissemination of the practical knowledge necessary to provide at minimal adequate care via telepsychiatry; most often, this focused on the technology and delivery of telepsychiatry learned by attendings in real time and passed to residents in a steady stream of e-mails. These e-mails were disseminated several times a day and at times included contradictory information given the speed with which guidelines were changing. The e-mails also included administrative updates, such as which states had suspended license requirements for telehealth visits and what billing codes should be used. The residency program instituted weekly virtual telepsychiatry supervision sessions led by an outpatient attending to provide residents the opportunity to seek supervision regarding the transition, but otherwise no formal telepsychiatry curriculum was initiated.

Given these challenging and unprecedented circumstances, we felt it would be useful to assess the PGY-3 resident experience with telepsychiatry.

\section{Surveying the Resident Experience}

A survey using Qualtrics was developed and distributed to 11 PGY-3 residents in our outpatient clinic. The Institutional Review Board reviewed the survey and determined it qualified for exempt status. An e-mail was distributed to residents that included the intent and goal of the survey, a statement reviewing that participation was anonymous and voluntary, and a link to access the survey. The e-mail was sent on June 5, 2020, with a reminder for non-completers sent on June 10, 2020.

The questions on the survey included the following:

1. When I began outpatient clinic, I had previously delivered psychiatric care through telepsychiatry (i.e., inpatient, emergency department, consults).

2. When I began outpatient clinic, I felt prepared to deliver care through telepsychiatry.
3. My clinical education was positively impacted by the implementation of telepsychiatry.

4. My patient care was positively impacted by the implementation of telepsychiatry.

5. PGY-3 clinic has given me enough experience to practice telepsychiatry without supervision in the future.

The first survey question prompted a yes/no response, and questions 2 through 5 asked residents to rate the strength of their opinions on a Likert scale of 1 to $5(1=$ strongly disagree, $2=$ disagree, $3=$ neutral, $4=$ agree, $5=$ strongly agree). The final question of the survey allowed residents to free text any feedback they had regarding the experience with transitioning to telepsychiatry in our outpatient clinic.

\section{Exploring Resident Feedback}

Nine of eleven PGY-3 residents responded to the survey (82\% response rate). Of respondents, 6 residents reported having exposure to telepsychiatry in other clinical settings prior to PGY-3 outpatient clinic. These telepsychiatry experiences likely occurred in our emergency department where the psychiatry emergency services team occasionally assigns residents to perform consults for an off-campus hospital via telemedicine. Given the infrequent and inconsistent nature of these consults, resident respondents still felt largely unprepared to deliver care through telepsychiatry, with an average rating of $2.78 \pm 0.92$.

The majority of residents felt that telepsychiatry positively impacted their clinical education experience (average rating of $4.33 \pm 0.67$ ), but less residents felt it had positively impacted their patient care (average rating of $3.78 \pm 1.23$ ). After providing telepsychiatry for approximately 2 months, 7 of 9 respondents strongly agreed they had enough experience to practice telepsychiatry without supervision in the future (average rating of $4.67 \pm 0.67$ ). Analysis with Stats iQ demonstrated a statistically significant relationship between the perception of telepsychiatry having a positive impact on clinical education and comfort with practicing telepsychiatry without supervision in the future ( $p=0.0359$, Chi-Squared Test). There was also a statistically significant improvement from retrospective to prospective ratings of preparation to practice telepsychiatry ( $p=0.002$, Mann-Whitney test) suggesting that residents perceived themselves to have transitioned from unprepared to prepared to deliver care via telepsychiatry.

Two residents included comments in the free text box soliciting feedback. One resident stated "I did not like performing telepsych visits over the phone. All new patient visits should be via video." The second resident provided program-specific feedback pertaining to their perception that they "could have learned more about the art of delivering psychiatric care over telephone/video". 


\section{Lessons Learned and Future Directions}

This survey captured the experience of 9 PGY-3 residents with the rapid transition from all face-to-face encounters to telepsychiatry for (nearly) all outpatient encounters. Given the crescendo of the pandemic and lack of formal telepsychiatry curriculum, there was minimal time for attendings, who were otherwise inexperienced with outpatient telepsychiatry themselves, to provide dedicated instruction to residents about evidence-based care and best practices. In addition, there were significant obstacles to implementing video visits (much preferred to telephone), rigorous supervision, and effective communication. Despite these challenges, the majority of residents went from feeling largely unprepared to deliver care via telepsychiatry to strongly agreeing that they are prepared to practice telepsychiatry without supervision.

While the telepsychiatry experience seemed to positively impact clinical education for the residents, patient care was not perceived to be as positively affected. The absence of attending expertise in telepsychiatry and lack of formal preparation for residents most likely contributed for this discrepancy. The need to prepare a formal curriculum to address the downstream patient care implications of telepsychiatry quickly emerged (i.e., managing difficult situations or maintaining patient-doctor rapport). The free text response by one of our residents stating they wanted to learn more about the art of delivering psychiatric care over telephone/video further supports this gap in the educational curriculum.

With some societal push to "return to normal," in-person appointments will be more accessible for patients in our resident clinic. However, a number of patients have expressed their strong preference to continue to receive services via telepsychiatry as long as they are covered by insurance policies. While the crisis accelerated the clinical education of residents, the need to develop multiple facets of telepsychiatry education for residents and attendings has become evident.

We have taken steps towards this by forming a multidisciplinary Telehealth Taskforce; a survey to assess faculty supervision needs will be disseminated, and the taskforce members are reviewing existing telepsychiatry literature to develop evidence-based practices. We are also generating a telepsychiatry lecture series focusing on the 3 competencies emphasized by Sunderji et al. in their narrative review of telepsychiatry in graduate medical education: technical, collaborative, and administrative [14]. This formal curriculum will review the now-established technological processes of outpatient telepsychiatry and add a more meaningful exploration into "the art" of delivering patient care via telepsychiatry and the administrative landscape of billing and compliance in telemedicine. To minimize confusion, communication with residents has been streamlined and consolidated to, at most, one summary e-mail a day.
Maintaining rigorous clinical supervision in the context of telepsychiatry will require attendings to develop new strategies. One initiative we are introducing will be observed interviews; an attending will conference into a resident telepsychiatry patient assessment quarterly to observe the appointment and will have dedicated time to provide individualized feedback to the resident after the appointment. The resident-attending pairings will be maintained over the course of the year to allow for a longitudinal perspective of individual resident's progress. We are implementing these initiatives at our program, but if telepsychiatry is here to stay, perhaps it is also time for the ACGME to mandate telepsychiatry curriculum for all residency programs.

The major limitation with this survey is the generalizability, as it was conducted with a small cohort of residents at one residency program. Other limitations include the lack of a preintervention survey and known limitation of using Likert scales in educational research [15]. There are several possible next steps within this area of education research, including exploring the experience of residents at other programs or even within other specialties. There will also be more opportunities for programs to compare and contrast the delivery of various telepsychiatry curricula, as COVID-19 will surely increase the number of residencies developing educational content for their trainees and faculty. Finally, there will be a need to explore the longitudinal impact on patient care and supervision models with the transition to telepsychiatry.

Acknowledgments I thank the residents and the outpatient resident clinic for their contribution to this project.

\section{Compliance with Ethical Standards}

Disclosures The author states that there is no conflict of interest.

Ethical Approval The research was approved by the Biomedical Institutional Review Board.

\section{References}

1. García-Lizana F, Muñoz-Mayorga I. Telemedicine for depression: a systematic review. Perspect Psychiatr Care. 2010;46:2.

2. Myers K, Cain S, Work Group on Quality Issues, American Academy of Child and Adolescent Psychiatry Staff. AACAP official action. Practice parameter for telepsychiatry with children and adolescents. J Am Acad Child Adolesc Psychiatry. 2008;47(12): 1468-83.

3. Szeftel R, Hakak R, Meyer S, Naqvi S, Sulman-Smith H, Delrahim $\mathrm{K}$, et al. Training psychiatric residents and fellows in a telepsychiatry clinic: a supervision model. Acad Psychiatry. 2008;32(5):393-9.

4. Hilty D, Luo J, Morache C, Marcelo D, Nesbitt T. Telepsychiatry: an overview for psychiatrists. CNS Drugs. 2002;16(8):527-48.

5. Brown F. Rural telepsychiatry. Psychiatr Serv. 1998;49(7):963-4.

6. O'Reilly R, Bishop J, Maddox K, Hutchinson L, Fisman M, Takhar J. Is telepsychiatry equivalent to face-to-face psychiatry? Results 
from a randomized controlled equivalence trial. Psychiatr Serv. 2007;58(6):836-43. https://doi.org/10.1176/ps.2007.58.6.836.

7. Saeed SA, Johnson TL, Bagga M, Glass O. Training residents in the use of telepsychiatry: review of the literature and a proposed elective. Psychiatry Q. 2016;88(2):271-83.

8. Hoffman P, Kane JM. Telepsychiatry education and curriculum development in residency training. Acad Psychiatry. 2014;39(1): $108-9$.

9. Jessica R, Oesterheld JR, Travers HP, et al. An introductory curriculum on telepsychiatry for psychiatric residents. Acad Psychiatry. 1999;23(3):166-7.

10. Glover JA, Williams E, Hazlett LJ, Campbell N. Connecting to the future: telepsychiatry in postgraduate medical education. Telemed e-Health. 2013;19(6):474-9.

11. Common Program Requirements - ACGME Home. https://www. acgme.org/Portals/0/PFAssets/ProgramRequirements/400 Psychiatry_2019.pdf?ver=2019-08-26-134127-827. Accessed $1 \overline{1}$ June 2020 .
12. Shore JH, Schneck CD, Mishkind MC. Telepsychiatry and the coronavirus disease 2019 pandemic - current and future outcomes of the rapid virtualization of psychiatric care. JAMA Psychiatry. 2020.

13. Lor U-P, Sousa J, Raja P, Mehrotra A, Barnett M, Huskamp HA. Suddenly becoming a "virtual doctor": experiences of psychiatrists transitioning to telemedicine during the COVID-19 pandemic. Psychiatr Serv. 2020. https://doi.org/10.1176/appi.ps.202000250.

14. Sunderji N, Crawford A, Jovanovic M. Telepsychiatry in graduate medical education: a narrative review. Acad Psychiatry. 2014;39(1):55-62.

15. Sullivan GM, Artino AR Jr. Analyzing and interpreting data from Likert-type scales. J Grad Med Educ. 2013;5:541-2.

Publisher's Note Springer Nature remains neutral with regard to jurisdictional claims in published maps and institutional affiliations. 